\title{
Maternal nutrient restriction in early pregnancy induces adipose tissue inflammation with a compensatory increase in adiponectin abundance in juvenile offspring
}

\author{
Don Sharkey, David Gardner, Michael Symonds and Helen Budge \\ University of Nottingham, Nottingham, UK
}

Early-pregnancy nutrition is often unbalanced and suboptimal, with excess carbohydrate, and more importantly can be lacking in adequate protein $^{(1,2)}$. This outcome may in part be secondary to nausea and vomiting that can affect 50-90\% of pregnancies in the first trimester. Reduced protein intake in early pregnancy can reduce birth weight and placental size and this outcome may predispose the offspring to later adult diseases such as diabetes and $\mathrm{CVD}^{(1)}$. Adiponectin, a potent insulin sensitiser secreted by adipocytes, decreases with increasing adiposity with a concurrent rise in adipose tissue inflammation induced by cytokines and macrophage accumulation. IL-18 is elevated in obese individuals and is known to suppress adiponectin expression ${ }^{(3)}$. The extent to which maternal nutrient restriction (NR) in early pregnancy can alter the inflammatory response in adipose tissue of non-obese juvenile offspring has not be studied.

The aims of the current study were to establish the effects of maternal NR during early pregnancy (0-30d) on subsequent adipose tissue dysregulation in juvenile offspring. It has previously been shown in sheep that late NR but not early NR offspring are insulin resistant and glucose intolerant at 1 year of age ${ }^{(4)}$.

Singleton-bearing sheep were randomised to receive either $100 \%$ metabolisable energy (ME) (controls; $n$ ) throughout pregnancy or $50 \%$ ME on days $0-30$ (early NR; $n$ 5). Offspring were reared by their mother until 3 months, weaned and fed at pasture until 1 year when they were humanely killed. Real-time PCR was used to measure the mRNA abundance of adiponectin, CD68 (macrophage marker), IL$18, \mathrm{TNF} \alpha$, monocyte chemoattractant protein-1 (MCP-1) and leptin in perirenal adipose tissue. Expression was normalised to 18s rRNA using the $2^{-\Delta \mathrm{ct}}$ method $^{(5)}$ and expressed relative to the control value. Results were analysed using Student's $t$ test and Pearson's correlation coefficient. All animal procedures were performed in accordance with UK legislation.

Birth weight (kg; controls, 4.3 (SE 0.3); NR, 4.5 (SE 0.2)), weight at 1 year (kg; controls, 50.7 (SE 3.7); NR, 49.8 (SE 4.3)) and relative fat mass (g/kg; controls, 7.7 (SE 1.3); NR, 9.7 (SE 0.6)) were not different between groups.

\begin{tabular}{|c|c|c|c|c|c|}
\hline \multirow[b]{2}{*}{ Gene* } & \multicolumn{2}{|c|}{ Controls } & \multicolumn{2}{|c|}{ Early NR } & \multirow[b]{2}{*}{$P$ value } \\
\hline & Mean & $\mathrm{SE}$ & Mean & $\mathrm{SE}$ & \\
\hline Adiponectin & 1.0 & 0.1 & 3.8 & 1.2 & 0.01 \\
\hline CD68 & 1.0 & 0.2 & 4.1 & 0.7 & 0.002 \\
\hline IL-18 & 1.0 & 0.2 & 2.5 & 1.1 & 0.009 \\
\hline $\mathrm{TNF} \alpha$ & 1.0 & 0.2 & 1.9 & 0.5 & 0.08 \\
\hline MCP-1 & 1.0 & 0.2 & 1.0 & 0.2 & 0.57 \\
\hline Leptin & 1.0 & 0.3 & 2.2 & 1.0 & 0.17 \\
\hline
\end{tabular}

Leptin expression and plasma levels ${ }^{(4)}$ were not different between the groups in keeping with their similar fat mass. Irrespective of maternal diet adiponectin abundance correlated with CD68 $\left(r^{2} 0.79 ; P<0.001\right)$, IL-18 $\left(r^{2} 0.72 ; P=0.001\right)$ and TNF $\alpha\left(r^{2} 0.66 ; P=0.001\right)$.

Despite early NR having no effect on birth weight, juvenile offspring show evidence of increased adipose tissue inflammation with an apparent compensatory increase in adiponectin expression. Adipose tissue inflammation is associated with the development of insulin resistance and obesity. These offspring show no evidence of insulin resistance and this outcome may reflect the compensatory increase in adiponectin thus delaying its onset. Further studies in later life could investigate whether this compensatory mechanism is overcome by on-going inflammation as a result of increasing IL-18 ultimately resulting in insulin resistance. This information may well have important implications for women with suboptimal nutrition in early pregnancy.

1. Godfrey K, Robinson S, Barker DJ et al. (1996) Br Med J 312, 410-414.

2. Moore VM, Davies MJ, Willson KJ et al. (2004) J Nutr 134, 1820-1826.

3. Chandrasekar B, Patel DN, Mummidi S et al. (2008) J Biol Chem 283, 4200-4209.

4. Gardner DS, Tingey K, Van Bon BW et al. (2005) Am J Physiol Regul Integr Comp Physiol 289, R947-R954.

5. Pfaffl MW (2001) Nucleic Acids Res 29(9), e45. 